Article

\title{
Analysis of the Connecting Effectiveness of the Interphase Zone on the Tensile Properties of Carbon Nanotubes (CNT) Reinforced Nanocomposite
}

\author{
Yasser Zare ${ }^{\circledR}$ and Kyong Yop Rhee *(i) \\ Department of Mechanical Engineering, College of Engineering, Kyung Hee University, Yongin 446-701, Korea; \\ y.zare@aut.ac.ir \\ * Correspondence: rheeky@khu.ac.kr; Tel.: +82-31-201-2565; Fax: +82-31-202-6693
}

Received: 26 March 2020; Accepted: 10 April 2020; Published: 13 April 2020

\begin{abstract}
The establishment of interphase region around nanoparticles accelerates the percolating of carbon nanotubes (CNT) in polymer nanocomposites reinforced with CNT (PCNT), due to the linking productivity of interphase district before the physical connecting of nanoparticles. Therefore, the interphase is an important character in the networks of CNT in PCNT. Here, a simulation study is presented to investigate the interphase connection in the mechanical possessions of PCNT including tensile modulus and strength. A number of models comprising Takayanagi, Ouali, Pukanszky and Callister are developed by the assumption of an interphase district in the CNT excluded volume. The advanced models depict the optimistic influences of reedy and lengthy CNT besides dense interphase on the stiffness and tensile power of nanocomposites. The Pukanszky calculations depict that the interphase strength plays a more noteworthy role in the nanocomposites strength compared to the CNT length.
\end{abstract}

Keywords: carbon nanotubes (CNT); polymer nanocomposites; interphase district; percolation onset; mechanical possessions

\section{Introduction}

The carbon nanotubes (CNT) as ideal nanoparticles can add high stiffness and good electrical conductivity to polymer matrices. Additionally, the nanoscale diameter and large aspect ratio of CNT significantly improve the general properties of polymer CNT nanocomposites (PCNT) [1-17]. The van der Waals attraction between nanotubes leads the aggregation/agglomeration in the nanocomposites, which reduces the filler surface expanse and interrupts the networking level [18-20]. Additionally, the interfacial communication/bond/area between the polymer medium and particles should be acceptable for an effective load transfer from the polymer matrix to CNT. So, it is important to facilitate the dispersion of nanoparticles at nanoscale and provide good interfacial properties through some techniques of compatibilizing [21,22].

The conductivity of PCNT is found after the filler percolation onset in which the nanofiller forms a network, which causes charge transport [23-25]. In addition to percolation threshold for conductivity in nanocomposites, an abrupt improvement of modulus/stiffness was found called a mechanical percolation [26-28]. The substantial level of shear modulus in the reinforced cellulose whisker films was attributed to mechanical percolation [29]. Therefore, the network of nanoparticles above a particular volume fraction as percolation onset causes a high increment in the mechanical possessions. The vast interfacial region around the nanoparticles and the numerous nanoparticles in a unit volume change the operative possessions of nanocomposites [30,31]. The interfacial area around the nanoparticles produces an intermediate phase as interphase in nanocomposite samples. 
The interphase is hardly considered by experimental measurements, owing to the minor extent and multifaceted style. Additionally, most available models on the mechanics cannot suppose the interphase effects $[18,32,33]$. Therefore, several new and developed models were suggested to study the interphase character in the mechanical behavior [34-36].

The interphase district around nanoparticles can facilitate the establishment of a networked structure in nanocomposites at a low filler portion [37]. This occurrence declines the percolation onset, due to the connection of interphase area. It was suggested that the interfacial communications between the polymer medium and particles manage the percolation of particles with a high aspect ratio and random orientation [38]. Nevertheless, the interphase percolation and its influences on the mechanical possessions have not been considered in the previous works on the nanocomposites. Even though the earlier articles have suggested the strengthening character of interphase, the interphase percolation was not clarified, well. The interphase can quicken the filler percolation making a novel tactic in the interphase and filler network in nanocomposites. In this article, the novel interphase percolation surrounding nanoparticles is defined to progress the available models for nanocomposites mechanical properties. This paper examines the effects of CNT size and interphase possessions on the tensile modulus and strength by Takayanagi, Ouali, Pukanszky and Callister models assuming interphase percolation. The outputs of this paper can conduct the investigators to understand the role of interphase in the percolating of nanoparticles. Moreover, the present article indicates the strengthening and percolating effects of interphase in nanocomposites.

\section{Equations and Developed Models}

The percolation onset in PCNT supposing the filler and interphase percolation was obtained [39] as:

$$
\phi_{p}=\frac{\pi R^{2} l+(4 / 3) \pi R^{3}}{\frac{32}{3} \pi(R+t)^{3}\left[1+\frac{3}{4}\left(\frac{l}{R+t}\right)+\frac{3}{32}\left(\frac{l}{R+t}\right)^{2}\right]}
$$

where " $R$ " and "l" show the CNT radius and length, correspondingly. In addition, " $t$ " denotes the interphase depth surrounding CNT.

Some authors [40] advanced the Takayanagi equation assuming the networking and dispersal of CNT exceeding percolation onset as:

$$
E=\frac{\phi_{N}\left(1-\phi_{f}\right) E_{f} E_{N}+\phi_{N}\left(\phi_{f}-\phi_{N}\right) E_{m} E_{N}+\left(1-\phi_{N}\right)^{2} E_{f} E_{m}}{\left(1-\phi_{f}\right) E_{f}+\left(\phi_{f}-\phi_{N}\right) E_{m}}
$$

where " $\phi_{f}$ " and " $\phi_{N}$ " show the volume portions of CNT and nets, correspondingly. Additionally, " $E_{\mathrm{f}}$ ", " $E_{\mathrm{N}}$ " and " $E_{\mathrm{m}}$ " denote the tensile moduli of detached nanofiller, net and polymer medium, correspondingly. The nonappearance of net $\left(\phi_{N}=0\right)$ condenses the advanced model to:

$$
E=\frac{E_{f} E_{m}}{\left(1-\phi_{f}\right) E_{f}+\phi_{f} E_{m}}
$$

The percentage of percolated CNT can be roughly expressed [41] by:

$$
f=1-\exp \left[-A\left(\frac{\phi_{f}}{\phi_{p}}-1\right)^{0.474}\right]
$$

where " $\mathrm{A}$ " is constant depending to the net level. The volume portion of filler net is also estimated as:

$$
\phi_{N}=\frac{f \phi_{f}}{1-(1-f) \phi_{f}} \cong f \phi_{f}
$$


By substituting Equations (4) and (5) into Equation (2), the tensile modulus of nanocomposites predicted by Takayanagi model is correlated to the percolation threshold.

Ouali et al. [42] also added the percolation onset to the reverse rule of mixtures as:

$$
E=\frac{\left(1-2 \psi+\psi \phi_{f}\right) E_{m} E_{f}+\left(1-\phi_{f}\right) \psi E_{f}^{2}}{\left(1-\phi_{f}\right) E_{f}+\left(\phi_{f}-\psi\right) E_{m}}
$$

where " $\psi$ " parameter depends on " $\phi_{p}$ " as:

$$
\psi=\phi_{f}\left(\frac{\phi_{f}-\phi_{p}}{1-\phi_{p}}\right)^{b}
$$

The " $\mathrm{b}$ " factor is a percolation exponent, which equals to 0.4 in 3D scheme [42]. Moreover, the relative modulus is defined as $\mathrm{E} / \mathrm{E}_{\mathrm{m}}$.

There are two known models for tensile strength/power of nanocomposites including Pukanszky and Callister. They do not include the " $\phi_{p}$ " parameter, but the aspect ratio of nanofiller $(\alpha)$ in these models can be related to " $\phi_{p}$ " above the percolation threshold. As a result, they are expressed by " $\phi_{p}$ " and the new form of " $\phi_{p}$ " (Equation (1)) develops them to show the interphase percolation.

Pukanszky [43] recommended a model for strength of composites as:

$$
\sigma_{R}=\frac{1-\phi_{f}}{1+2.5 \phi_{f}} \exp \left(B \phi_{f}\right)
$$

where " $\sigma_{\mathrm{R}}$ " is relative strength as $\sigma_{\mathrm{c}} / \sigma_{\mathrm{m}} ; "{ }_{\mathrm{c}}$ " and " $\sigma_{\mathrm{m}}$ " show the tensile strength of composite and polymer medium, correspondingly. In addition, " $\mathrm{B}$ " as an interfacial factor displays the extent of stress flowing between polymer medium and filler. This model has been acceptably applied for strength of polymer nanocomposites [44,45].

The " $B$ " parameter depends on the thickness and strength of interphase by:

$$
B=\left(1+A_{c} d_{f} t\right) \ln \left(\frac{\sigma_{i}}{\sigma_{m}}\right)
$$

where " $A_{\mathrm{c}}$ " and " $d_{\mathrm{f}}$ " show the specific surface area and density of nanofiller, in that order. Additionally, " $\sigma_{\mathrm{i}}$ " is interphase strength. " $A_{\mathrm{c}}$ " for cylindrical rods such as CNT is formulated by:

$$
A_{c}=\frac{A}{m}=\frac{A}{d_{f} V} \cong \frac{2 \pi R l}{d_{f} \pi R^{2} l}=\frac{2}{d_{f} R}=\frac{4 \alpha}{d_{f} l}
$$

where " $A$ " and " $m$ " show the filler surface area and mass, correspondingly. In addition, $\alpha=1 / d$, where " $d$ " is the diameter of particles.

Chatterjee [41] suggested a simple equation between " $\phi_{p}$ " and " $\alpha$ " as:

$$
\phi_{p} \approx \frac{1}{\alpha}
$$

By replacing of " $\alpha$ " from Equation (11) into Equation (10), " $A_{\mathrm{c}}$ ” can be given by:

$$
A_{c}=\frac{4}{d_{f} l \phi_{p}}
$$

By substituting of " $A_{\mathrm{c}}$ " from Equation (12) into Equation (9), " $B$ " is stated by percolation onset as:

$$
B=\left(1+\frac{4}{l \phi_{p}} t\right) \ln \left(\frac{\sigma_{i}}{\sigma_{m}}\right)
$$


When " $B$ " from above equation is considered in Equation (8), the relative strength is estimated by:

$$
\sigma_{R}=\frac{1-\phi_{f}}{1+2.5 \phi_{f}} \exp \left(\left(1+\frac{4}{l \phi_{p}} t\right) \ln \left(\frac{\sigma_{i}}{\sigma_{m}}\right) \phi_{f}\right)
$$

Callister [46] also projected the yield strength of polymer composites by interfacial properties as:

$$
\sigma_{R}=1+\left(\frac{\alpha s}{\sigma_{m}}-1\right) \phi_{f}
$$

where " $s$ " denotes the interfacial stress transferring. Even if this model was originally proposed for short fiber composites, it can be effectively used for polymer nanocomposites [47,48].

The Callister model can be expanded by percolation onset (Equation (11)) as:

$$
\sigma_{R}=1+\left(\frac{s}{\phi_{p} \sigma_{m}}-1\right) \phi_{f}
$$

which simply connects the nanocomposites strength to percolation onset. When the " $\phi_{p}$ " from Equation (1) is replaced into Equation (16), the relative strength above the percolation onset is represented as:

$$
\sigma_{R}=1+\left(\frac{s \frac{32}{3} \pi(R+t)^{3}\left[1+\frac{3}{4}\left(\frac{l}{R+t}\right)+\frac{3}{32}\left(\frac{l}{R+t}\right)^{2}\right]}{\left[\pi R^{2} l+(4 / 3) \pi R^{3}\right] \sigma_{m}}-1\right) \phi_{f}
$$

which explicitly correlates the strength of nanocomposites to matrix, nanoparticles and interphase properties accounting for the interphase percolation.

\section{Results and Discussion}

The expressed models were utilized to investigate the filler and interphase belongings in the mechanical properties of nanocomposites assuming the interphase percolation. In this regard, the mechanical properties were studied as a function of many factors such as the particle size, interphase thickness and strength as well as network modulus.

Figure 1 revealed the " $R$ " and " $t$ " roles in the tensile modulus of nanocomposite based on the advanced Takayanagi model and interphase percolation at $E_{\mathrm{m}}=2 \mathrm{GPa}, \phi_{f}=0.02, E_{\mathrm{f}}=1000 \mathrm{GPa}$, $l=5 \mu \mathrm{m}, A=0.02$ and $E_{\mathrm{N}}=2000 \mathrm{GPa}$. The high ranges of modulus were acquired by reedy nanoparticles and dense interphase. As observed, $E_{\mathrm{R}}=3.2$ was reported at $R=10 \mathrm{~nm}$ and $t=30 \mathrm{~nm}$. However, the high levels of " $R$ " and small values of " $t$ " negligibly progressed the modulus. Therefore, the modulus of nanocomposites inversely related to the radius of nanoparticles, while the interphase thickness caused a positive effect on the modulus.

The thin nanotubes could produce a large level of surface part, which grew the interfacial area. As known, the big interfacial area at the polymer-nanoparticles interface promoted the interfacial interaction and improved the modulus. Additionally, the small nanoparticles caused the robust interfacial communications with the polymer medium, owing to the analogous scopes of polymer chains and nanofillers [49]. So, the thin nanotubes caused the sturdy connections amid polymer medium and nanoparticles, which increased the modulus. On the other hand, a denser interphase yielded a superior modulus demonstrating the positive effect of interphase percolation on the modulus. A thick interphase facilitated the connection between interfacial region and formation of the filler network without the physical links between nanoparticles. Therefore, a thicker interphase caused a better network, which caused a better modulus. Nevertheless, thick nanoparticles made a low aspect ratio and small interfacial area. In addition, a thin interphase delayed the formation of a network in the sample, which insignificantly increased the modulus. Accordingly, the thick nanotubes and thin interphase could not enhance the modulus of nanocomposites. 
a

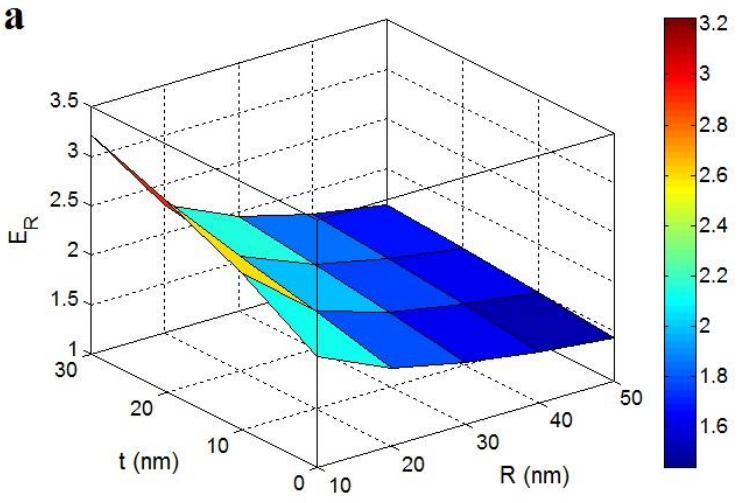

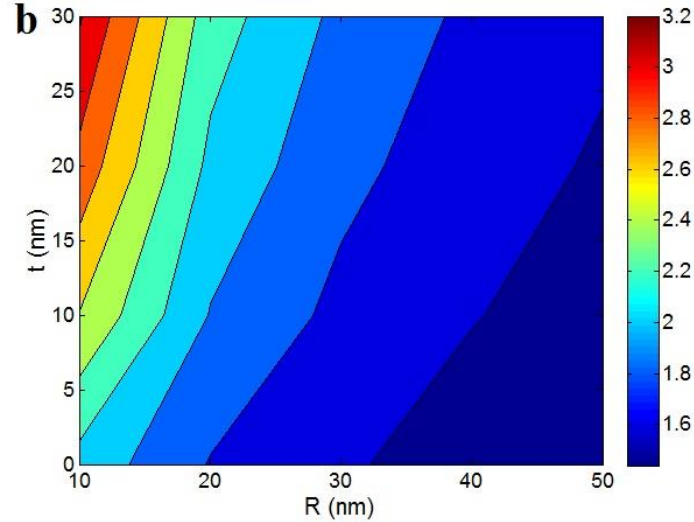

Figure 1. (a) $3 \mathrm{D}$ and (b) contour designs to show the relative modulus at dissimilar " $R$ " and " $t$ " ranges according to advanced Takayanagi model $\left(E_{\mathrm{m}}=2 \mathrm{GPa}, \phi_{f}=0.02, E_{\mathrm{f}}=1000 \mathrm{GPa}, l=5 \mu \mathrm{m}, A=0.02\right.$ and $\left.E_{\mathrm{N}}=2000 \mathrm{GPa}\right)$.

Figure 2 also shows the " $l$ " and " $E_{\mathrm{N}}$ " effects on the relative modulus by an advanced Takayanagi model at $E_{\mathrm{m}}=2 \mathrm{GPa}, \phi_{f}=0.02, R=10 \mathrm{~nm}, E_{\mathrm{f}}=1000 \mathrm{GPa}, \mathrm{A}=0.02$ and different thicknesses of interphase. The modulus shows a low level at the poor values of " $E_{\mathrm{N}}$ ". However, the uppermost modulus was calculated by the top levels of " $l$ " and " $E_{\mathrm{N}}$ ". About $50 \%$ improvement in modulus was observed at $E_{\mathrm{N}}<1200 \mathrm{GPa}$ at $t=5 \mathrm{~nm}$ (Figure 2a), but nearly $400 \%$ growth in modulus was observed at $l=7000 \mathrm{~nm}$ and $E_{\mathrm{N}}=5000 \mathrm{GPa}$ at the same interphase. These observations show the desirable effects of filler length and network modulus in the stiffness of nanocomposites. Clearly, lengthy CNT with a high aspect relation and large interfacial area yielded a robust interfacial interaction with polymer media. These factors facilitated the stress moving, which grew the nanocomposites modulus. Additionally, a strong network of nanoparticles could stand the big stress, which caused a high stiffness in the nanocomposite. The polymer matrix with low stiffness could not tolerate the stress loading, but the network of nanoparticles with extraordinary modulus could significantly reinforce the polymer matrix against the high stress.
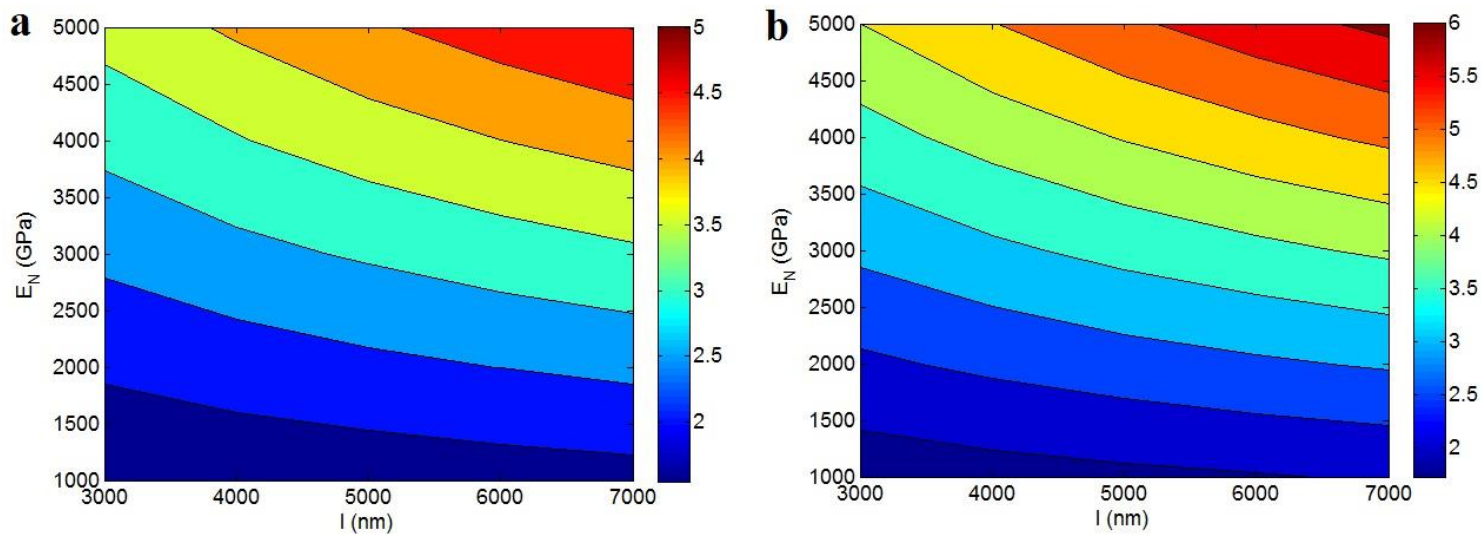

Figure 2. Contour plots for " $l$ " and " $E_{\mathrm{N}}$ " roles in the relative modulus by advanced Takayanagi model at (a) $t=5 \mathrm{~nm}$ and (b) $t=15 \mathrm{~nm}\left(E_{\mathrm{m}}=2 \mathrm{GPa}, \phi_{f}=0.02, R=10 \mathrm{~nm}, E_{\mathrm{f}}=1000 \mathrm{GPa}, A=0.02\right)$.

Figure $2 \mathrm{~b}$ displays the " $l$ " and " $E_{\mathrm{N}}$ " effects on the relative modulus at a thicker interphase as $t=15 \mathrm{~nm}$. The similar roles of these factors in the modulus were also obserevd in this illustration, but the level of modulus increased when the interphase thickness rose. At the same levels of $l=7000 \mathrm{~nm}$ and $E_{\mathrm{N}}=5000 \mathrm{GPa}$, the highest " $E_{\mathrm{R}}$ " at $t=5 \mathrm{~nm}$ was obtained as 5 , while the " $E_{\mathrm{R}}$ " increased to 6 at $t=15 \mathrm{~nm}$. As a result, the formation of a denser interphase caused a greater modulus, due to the better properties of network produced by interphase percolation. 
Figure 3 demonstrates the " $R$ " and " $t$ " influences on the relative modulus by the Ouali model at $E_{\mathrm{m}}=2 \mathrm{GPa}, \phi_{f}=0.02, E_{\mathrm{f}}=1000 \mathrm{GPa}$ and $l=5 \mu \mathrm{m}$. The relative modulus of about 3 was predicted at low " $R$ " and high " $t$ ", while the smallest modulus $\left(E_{R}=2.65\right)$ was detected at high " $R$ " and minor " $t$ ". Hence, the relative modulus was inversely and straightly linked with the radius of nanoparticle and thickness of interphase, respectively. These predictions were similar to those of developed Takayanagi model, which confirmed the parameters' roles in the total stiffness.
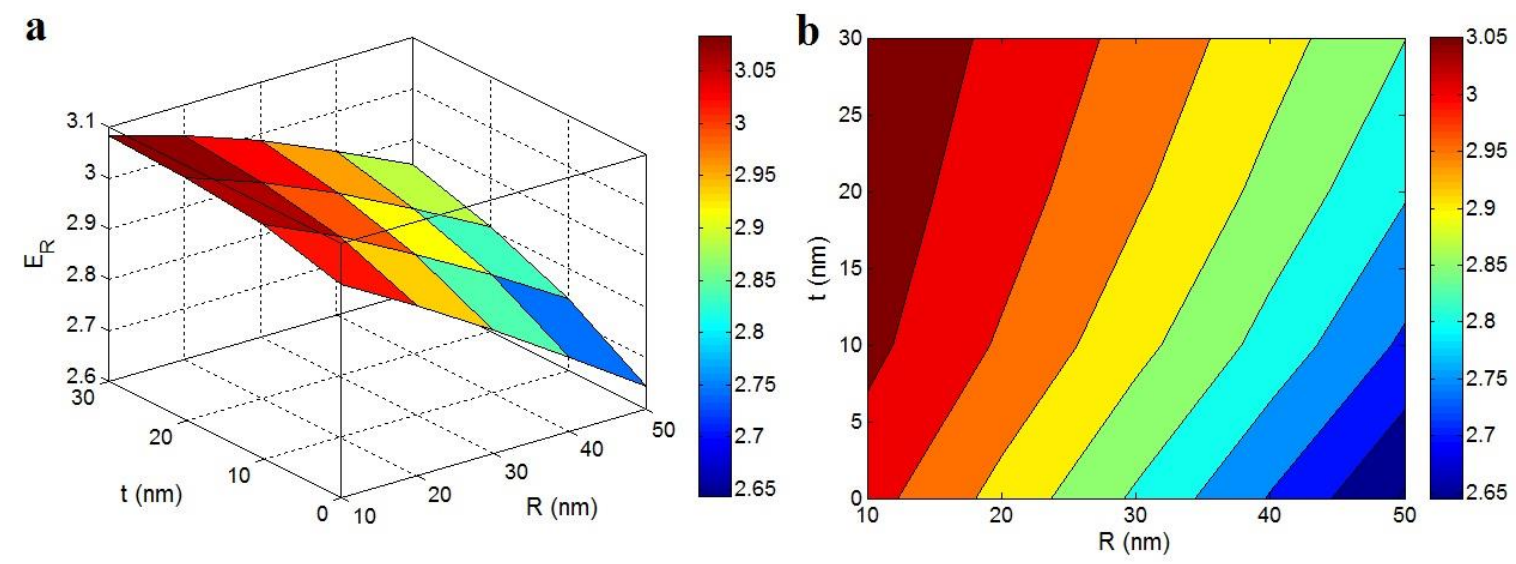

Figure 3. The relative modulus as a function of " $R$ " and " $t$ " terms by the Ouali model: (a) $3 \mathrm{D}$ and (b) contour plots.

The thin nanotubes show the acceptable dispersion of nanoparticles at the nanoscale in the polymer matrix, because some unwanted occurrences such as an accumulation increase of the radius of particles [50]. On the other hand, a dense interphase can hasten the development of filler net in the nanocomposites, which largely progresses the modulus. Consequently, the Ouali model suitably shows the " $R$ " and " $t$ " roles in the relative modulus accounting the percolating of interphase. In addition to the percolating effect of interphase, the good reinforcing efficiency of interphase has been well reported [51,52], due to its higher modulus compared to polymer matrix.

Figure 4 illustrates the " $R$ " and " $t$ " influences on the relative strength according to Pukanszky model at $\sigma_{\mathrm{m}}=40 \mathrm{MPa}, \phi_{f}=0.02, \sigma_{\mathrm{i}}=100 \mathrm{MPa}$ and $l=5 \mu \mathrm{m}$. This model predicted $\sigma_{\mathrm{R}}<1$ (the poorer strength of the nanocomposite than the polymer matrix) at high " $R$ " and small values of " $t$ ". This occurrence demonstrates that the strength of nanocomposites did not improve by big nanoparticles and a thin interphase. However, the improvement of strength started by decreasing in " $R$ " and increasing in " $t$ ". The highest strength based on the Pukanszky model as $\sigma_{R}=2.4$ was observed by the smallest nanoparticles and the thickest interphase. Hence, the extents of both particles and interphase significantly changed the nanocomposites strength.

Thin nanoparticles provided a high strengthening role in the nanocomposites, because they made a huge specific surface extent (Equation (10)), which significantly increased the interfacial communications. It was indicated that the high surface area of nanoparticles and strong interfacial interaction/adhesion are essential to realize an extraordinary strength in the nanocomposites $[44,53,54]$. On the other hand, a dense interphase caused a high excluded volume and low percolation onset in the nanocomposites, which accelerated the formation of net by large interfacial region. As a result, the strength of nanocomposites improved by the assumption of interphase percolation in the nanocomposites. Beside the percolation of interphase, a thick interphase (great $t$ ) significantly improved the strength of nanocomposites by its strengthening nature as shown in the Pukanszky model (Equation (14)). 

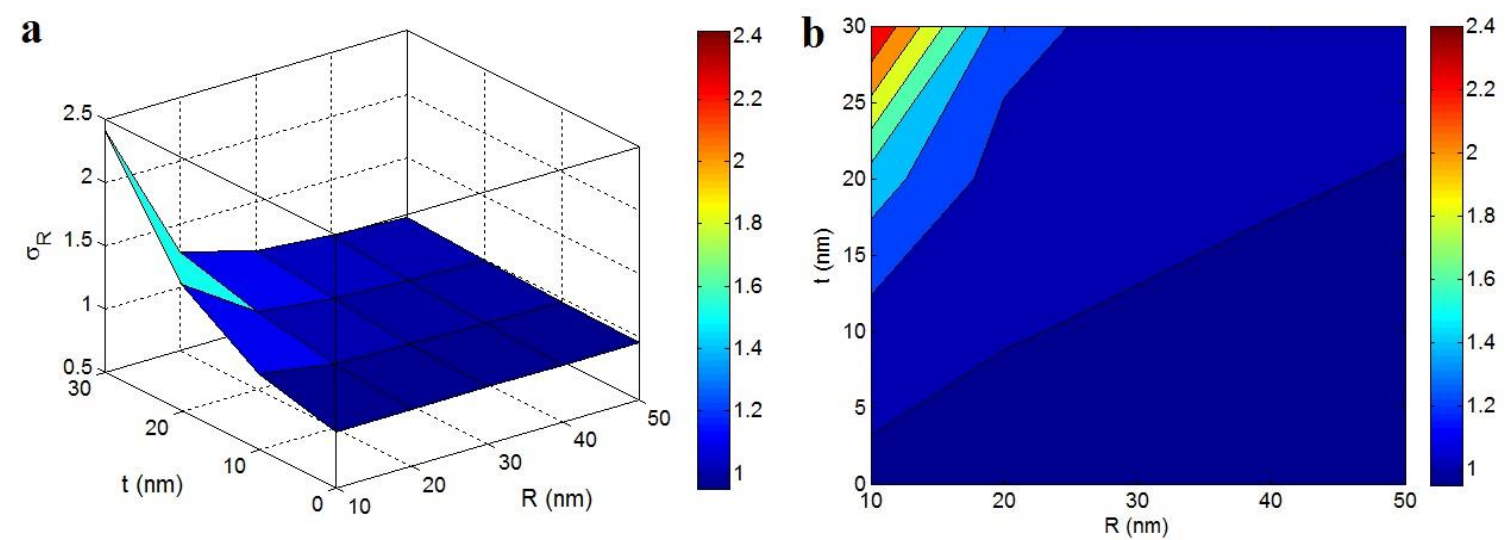

Figure 4. The influences of " $R$ " and " $t$ " terms on the relative strength assuming the percolating interphase based on the Pukanszky model at (a) 3D and (b) contour illustrations $\left(\sigma_{\mathrm{m}}=40 \mathrm{MPa}\right.$, $\phi_{f}=0.02, \sigma_{\mathrm{i}}=100 \mathrm{MPa}$ and $\left.l=5 \mu \mathrm{m}\right)$.

Figure 5 also displays the Pukanszky predictions at changed " $l$ " and " $\sigma_{\mathrm{i}}$ " levels $\left(\sigma_{\mathrm{m}}=40 \mathrm{MPa}\right.$, $\phi_{f}=0.02, R=10 \mathrm{~nm}$ and $t=5$ and $\left.15 \mathrm{~nm}\right)$. As observed, the " $\sigma_{\mathrm{i}}$ " parameter played a dominant character in the nanocomposites strength. The level of interphase strength directly controlled the strength for nanocomposites, while the CNT length did not affect the nanocomposites power in Figure $5 \mathrm{a}(t=5 \mathrm{~nm})$. Consequently, the role of interphase strength in the nanocomposites strength was more important than that of the nanotubes length. It demonstrated that the properties of interphase played a chief character in the strength. However, it should be noted that the poor levels of " $\sigma_{i}$ " smaller than $125 \mathrm{MPa}$ could not progress the relative strength. Accordingly, the deprived strength of samples might be related to the low strength of interphase.
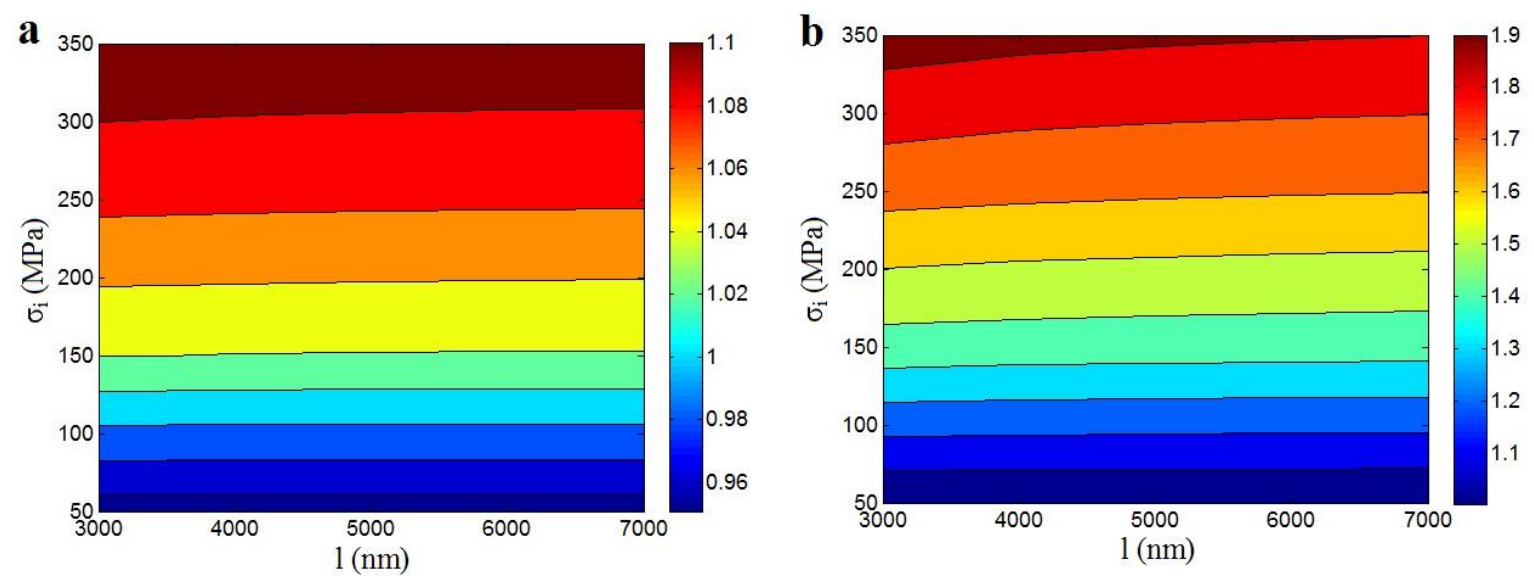

Figure 5. The predictions of Pukanszky model at different levels of " $l$ " and " $\sigma_{\mathrm{i}}$ " factors at $\sigma_{\mathrm{m}}=40 \mathrm{MPa}$, $\phi_{f}=0.02, R=10 \mathrm{~nm}$ and (a) $t=5 \mathrm{~nm}$ and (b) $t=15 \mathrm{~nm}$.

Figure $5 \mathrm{~b}$ also displays the important character of interphase power in the relative strength, but the nanotubes length can slightly affect the strength at the high ranks of " $\sigma_{\mathrm{i}}$ ". Furthermore, the highest level of relative strength was obtained as 1.9 at $\sigma_{\mathrm{i}}=350$ and $t=15 \mathrm{~nm}$ (Figure 5b), while the highest relative strength of 1.1 was obtained at the same interphase strength and $t=5 \mathrm{~nm}$ (Figure $5 \mathrm{a}$ ). As a result, the thickness of interphase had a positive effect on the tensile power. As mentioned, the interphase could decrease the percolation volume fraction of nanoparticles in the specimens by contacting of interphase area around the nanoparticles without the direct joining of nanoparticles. Additionally, the interphase induced a strengthening role in the nanocomposites, as reflected in the 
literature $[27,55]$. Therefore, the interphase undoubtedly promoted the strength of nanocomposites through strengthening effect and altering the percolation point.

The influences of nanoparticles radius and interphase thickness on the relative strength are shown in Figure 6 utilizing the Callister model and interphase percolation. The Callister model calculates the worst strength of nanocomposites at high " $R$ " levels. As indicated, the high levels of " $R$ " decline the reinforcing effect of nanoparticles through decreasing the specific surface area and weakening the interfacial interaction. As detected in Figure 6, the uppermost strength was seen at the least " $R$ " and the highest " $t$ ". A great level of " $t$ " displays the robust interfacial interaction/adhesion [18,43]. Moreover, a thick interphase easily produced a large net at low concentration of nanofiller. Consequently, the interphase is a vital factor in the possessions of nanocomposites.
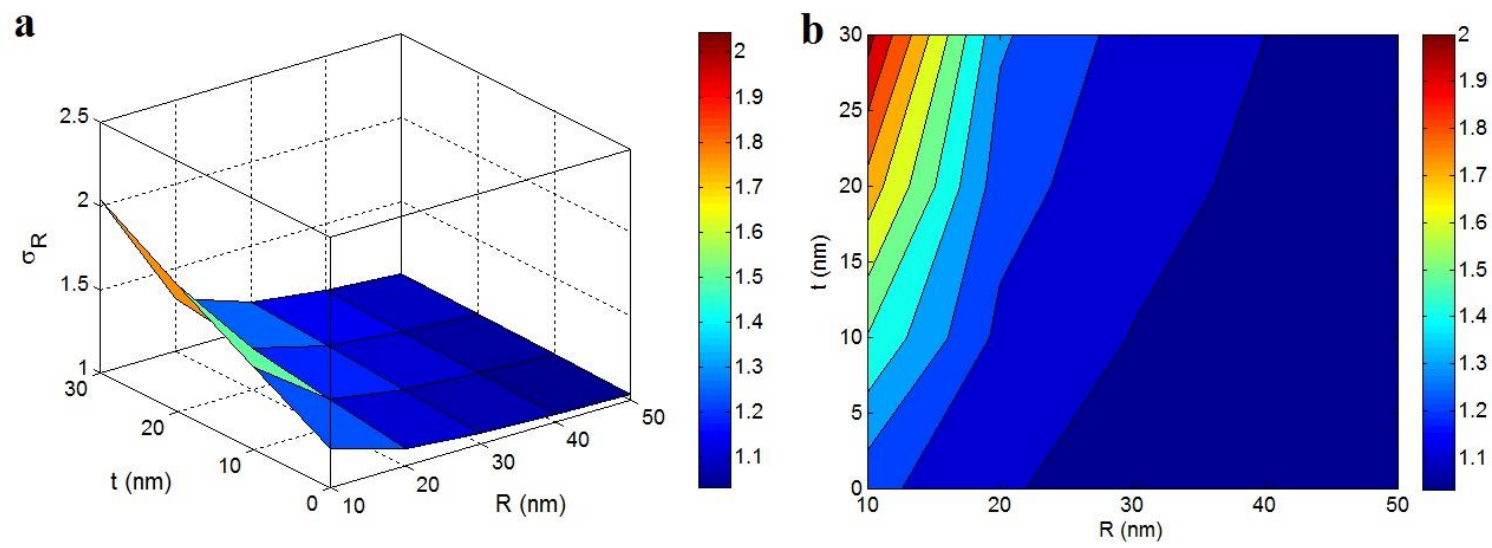

Figure 6. (a) $3 \mathrm{D}$ and (b) contour schemes for the influences of " $R$ " and " $t$ " on the relative strength expending the Callister model $\left(\sigma_{\mathrm{m}}=40 \mathrm{MPa}, \phi_{f}=0.02, s=1 \mathrm{MPa}\right.$ and $\left.l=5 \mu \mathrm{m}\right)$.

Figure 7 also illustrates the " 1 " and " $\mathrm{s}$ " roles in the relative strength based on Equation (17) at $\sigma_{\mathrm{m}}=40 \mathrm{MPa}, \phi_{f}=0.02, R=10 \mathrm{~nm}$ and $t=5$ and $15 \mathrm{~nm}$. The highest relative strength is acquired by the uppermost levels of " $l$ " and " $s$ " factors. Therefore, these parameters positively control the relative strength. It is expected, since the nanotubes length as a material parameter determines the levels of aspect ratio and interfacial area. In addition, a high " $l$ " parameter increased the level of excluded volume, which decreased the percolation threshold of nanoparticles in the nanocomposites (see Equations (6) and (7)). Furthermore, the " $s$ " parameter shows the stress transfer as a function of interfacial interaction/adhesion at the interface. Clearly, a high " $s$ " creates an excessive strength in the nanocomposites.
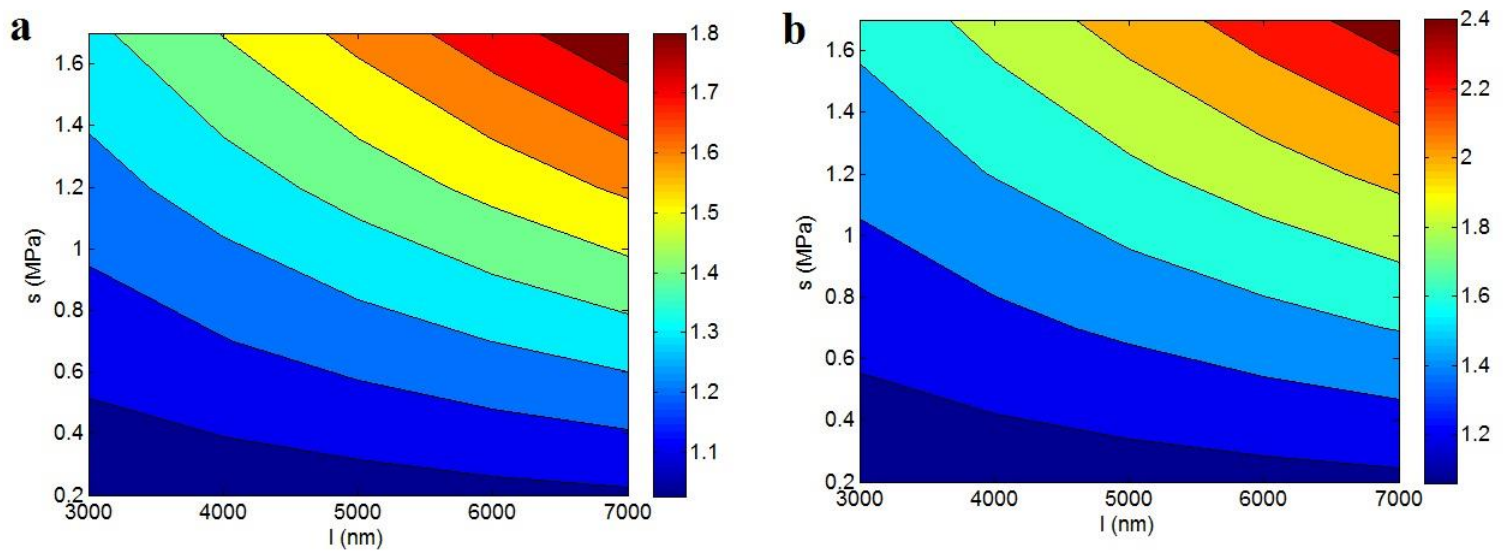

Figure 7. The roles of " $l$ " and " $s$ " parameters in the relative strength established by the Callister model: (a) $t=5 \mathrm{~nm}$ and (b) $t=15 \mathrm{~nm}$. 
The "l" and "s" parameters analogously changed the strength of nanocomposites in Figure 7a,b, but the level of improved strength was different depending on the interphase thickness. The relative strength improved from 1.03 to 1.8 in Figure $7 \mathrm{a}$ at $t=5 \mathrm{~nm}$, while it grew from 1.06 to 2.4 in Figure $7 \mathrm{~b}$ at $t=15 \mathrm{~nm}$. Therefore, a more interphase thickness produced better strength in the nanocomposites. According to the mentioned remarks, the interphase advanced the strengthening effect and percolating of nanofillers, which increased the strength of nanocomposites.

Figure 8 shows the experimental and theoretical relative modulus by an advanced Takayanagi model (Equation (2)) for epoxy/multi wall CNT (MWCNT) nanocomposites [56]. $E_{\mathrm{m}}=0.52 \mathrm{GPa}$ as well as average $R=25 \mathrm{~nm}$ and $l=50 \mu \mathrm{m}$ were reported in that paper. In addition, $E_{\mathrm{f}}=1000 \mathrm{GPa}$ and $A=0.02$ were considered for calculations. As observed, the experimental and theoretical data show good agreement at all CNT concentrations. This agreement confirms the predictability of the advanced Takayanagi model assuming a percolation threshold. In fact, this model considered the roles of interphase thickness, network modulus and percolation onset in the modulus of nanocomposites. Accordingly, $t=5 \mathrm{~nm}$ and $E_{\mathrm{N}}=2100 \mathrm{GPa}$ were obtained producing the percolation threshold of 0.0004 for this sample. These results indicate that this epoxy/MWCNT sample included the interphase region and CNT network based on the suggested equations.

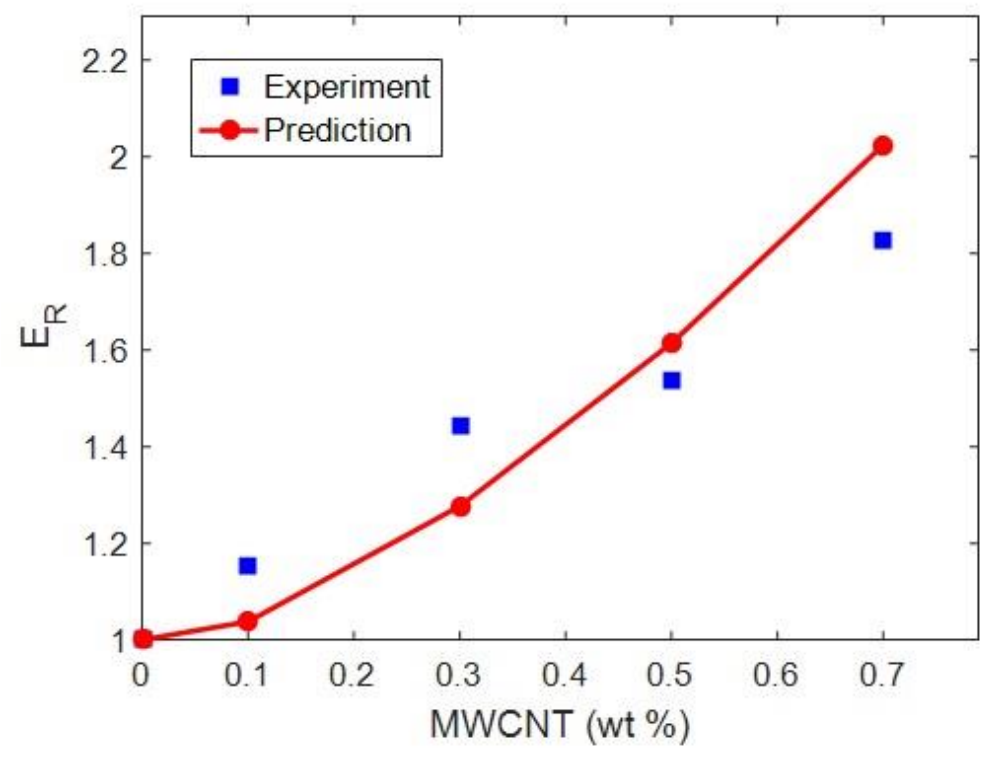

Figure 8. Experimental and theoretical relative modulus by the advanced Takayanagi model (Equation (2) for epoxy/MWCNT nanocomposites [56].

\section{Conclusions}

The interphase district was assumed in the percolation onset of the nanofiller to develop the Takayanagi, Ouali, Pukanszky and Callister models for tensile properties. These models investigated the effects of some factors attributed to nanoparticles and interphase on the tensile modulus and strength. Both Takayanagi and Ouali models predicted the high ranges of relative modulus by reedy nanoparticles and dense interphase, because reedy CNT cause a big interfacial area and robust interfacial communications with polymer medium. Moreover, a thick interphase facilitates the formation of filler net at lower nanofiller concentration without the filler physical links. The Takayanagi model also revealed the optimistic effects of nanotubes length and net modulus on the tensile modulus. Pukanszky and Callister models predicted the poor strength for nanocomposites at high levels of nanoparticles radius and small values of interphase thickness. However, the highest strength was observed by the smallest nanoparticles and the thickest interphase. The interphase strength played a more significant role in the nanocomposites strength in comparison to CNT length supposing the 
Pukanszky calculations. Conclusively, the tensile belongings of nanocomposites generally increase through the reinforcing effect of interphase and decreasing the percolation point by interphase area.

Author Contributions: Formal analysis, Y.Z.; Methodology, Y.Z.; Project administration, K.Y.R.; Supervision, K.Y.R.; Writing—original draft, Y.Z.; Writing—review and editing, K.Y.R. All authors have read and agreed to the published version of the manuscript.

Funding: This research received no external funding.

Conflicts of Interest: The authors declare no conflict of interest.

\section{References}

1. Zare, Y.; Rhee, K.Y. Prediction of loss factor $(\tan \delta)$ for polymer nanocomposites as a function of yield tress, relaxation time and the width of transition region between Newtonian and power-law behaviors. J. Mech. Behav. Biomed. Mater. 2019, 96, 136-143. [CrossRef] [PubMed]

2. Liu, S.; Wu, G.; Chen, X.; Zhang, X.; Yu, J.; Liu, M.; Zhang, Y.; Wang, P. Degradation Behavior In Vitro of Carbon Nanotubes (CNTs)/Poly (lactic acid)(PLA) Composite Suture. Polymers 2019, 11, 1015. [CrossRef] [PubMed]

3. Otaegi, I.; Aranburu, N.; Iturrondobeitia, M.; Ibarretxe, J.; Guerrica-Echevarría, G. The Effect of the Preparation Method and the Dispersion and Aspect Ratio of CNTs on the Mechanical and Electrical Properties of Bio-Based Polyamide-4, 10/CNT Nanocomposites. Polymers 2019, 11, 2059. [CrossRef] [PubMed]

4. Zagho, M.; AlMaadeed, M.; Majeed, K. Thermal properties of TiO2NP/CNT/LDPE hybrid nanocomposite films. Polymers 2018, 10, 1270. [CrossRef]

5. Wang, X.; Wang, H.; Liu, B. Carbon Nanotube-Based Organic Thermoelectric Materials for Energy Harvesting. Polymers 2018, 10, 1196. [CrossRef]

6. Eltayeb, N.E.; Khan, A. Design and Preparation of a New and Novel Nanocomposite with CNTs and Its Sensor Applications. J. Mater. Res. Technol. 2019, 8, 2238-2246. [CrossRef]

7. Rao, Y.A.; Ramji, K.; Rao, P.S.; Srikanth, I. Effect of A-MWCNTs and ETBN toughener on impact, compression and damping properties of carbon fiber reinforced epoxy composites. J. Mater. Res. Technol. 2019, 8, 896-903. [CrossRef]

8. Wang, C.-F.; Wang, W.-N.; Lin, C.-H.; Lee, K.-J.; Hu, C.-C.; Lai, J.-Y. Facile Fabrication of Durable Superhydrophobic Films from Carbon Nanotube/Main-Chain Type Polybenzoxazine Composites. Polymers 2019, 11, 1183. [CrossRef]

9. Wu, G.; Gu, Y.; Hou, X.; Li, R.; Ke, H.; Xiao, X. Hybrid Nanocomposites of Cellulose/Carbon-Nanotubes/ Polyurethane with Rapidly Water Sensitive Shape Memory Effect and Strain Sensing Performance. Polymers 2019, 11, 1586. [CrossRef]

10. Wang, J.; Cao, C.; Chen, X.; Ren, S.; Chen, Y.; Yu, D.; Chen, X. Orientation and Dispersion Evolution of Carbon Nanotubes in Ultra High Molecular Weight Polyethylene Composites under Extensional-Shear Coupled Flow: A Dissipative Particle Dynamics Study. Polymers 2019, 11, 154. [CrossRef]

11. Keshtkar, M.; Mehdipour, N.; Eslami, H. Thermal Conductivity of Polyamide-6, 6/Carbon Nanotube Composites: Effects of Tube Diameter and Polymer Linkage between Tubes. Polymers 2019, 11, 1465. [CrossRef] [PubMed]

12. Zare, Y.; Rhee, K.Y. Following the morphological and thermal properties of PLA/PEO blends containing carbon nanotubes (CNTs) during hydrolytic degradation. Compos. Part B Eng. 2019, 175, 107132. [CrossRef]

13. Zare, Y.; Rhee, K.Y. Effects of interphase regions and filler networks on the viscosity of PLA/PEO/carbon nanotubes biosensor. Polym. Compos. 2019, 40, 4135-4141. [CrossRef]

14. Zare, Y.; Rhee, K.Y.; Park, S.J. Simple model for hydrolytic degradation of poly (lactic acid)/poly (ethylene oxide)/carbon nanotubes nanobiosensor in neutral phosphate-buffered saline solution. J. Biomed. Mater. Res. Part A 2019, 107, 2706-2717. [CrossRef] [PubMed]

15. Rostami, A.; Moosavi, M.I. High-performance thermoplastic polyurethane nanocomposites induced by hybrid application of functionalized graphene and carbon nanotubes. J. Appl. Polym. Sci. 2019, 137, 48520. [CrossRef] 
16. Rostami, A.; Vahdati, M.; Alimoradi, Y.; Karimi, M.; Nazockdast, H. Rheology provides insight into flow induced nano-structural breakdown and its recovery effect on crystallization of single and hybrid carbon nanofiller filled poly (lactic acid). Polymer 2018, 134, 143-154. [CrossRef]

17. Rostami, A.; Vahdati, M.; Nazockdast, H. Unraveling the localization behavior of MWCNTs in binary polymer blends using thermodynamics and viscoelastic approaches. Polym. Compos. 2018, 39, 2356-2367. [CrossRef]

18. Hassanzadeh-Aghdam, M.K.; Mahmoodi, M.J.; Ansari, R. Creep performance of CNT polymer nanocomposites-An emphasis on viscoelastic interphase and CNT agglomeration. Compos. Part B Eng. 2019, 168, 274-281. [CrossRef]

19. Hassanzadeh-Aghdam, M.; Ansari, R.; Mahmoodi, M.; Darvizeh, A. Effect of nanoparticle aggregation on the creep behavior of polymer nanocomposites. Compos. Sci. Technol. 2018, 162, 93-100. [CrossRef]

20. Zare, Y.; Rhee, K.Y. A Simulation Work for the Influences of Aggregation/Agglomeration of Clay Layers on the Tensile Properties of Nanocomposites. JOM 2019, 71, 3989-3995. [CrossRef]

21. Abdolmaleki, A.; Mallakpour, S.; Borandeh, S. Amino acid-functionalized multi-walled carbon nanotubes for improving compatibility with chiral poly (amide-ester-imide) containing l-phenylalanine and l-tyrosine linkages. Appl. Surf. Sci. 2013, 287, 117-123. [CrossRef]

22. Orgilés-Calpena, E.; Arán-Aís, F.; Torró-Palau, A.M.; Orgilés-Barceló, C. Chemical functionalization and dispersion of carbon nanofibers in waterborne polyurethane adhesives. J. Adhes. 2013, 89, 174-191.

23. Zare, Y.; Rhee, K.Y. Calculation of the Electrical Conductivity of Polymer Nanocomposites Assuming the Interphase Layer Surrounding Carbon Nanotubes. Polymers 2020, 12, 404. [CrossRef] [PubMed]

24. Zare, Y.; Rhee, K.Y.; Park, S.-J. A developed equation for electrical conductivity of polymer carbon nanotubes (CNT) nanocomposites based on Halpin-Tsai model. Results Phys. 2019, 14, 102406. [CrossRef]

25. Zare, Y.; Rhee, K.Y. The effective conductivity of polymer carbon nanotubes (CNT) nanocomposites. J. Phys. Chem. Solids 2019, 131, 15-21. [CrossRef]

26. Zare, Y.; Garmabi, H.; Rhee, K.Y. Prediction of complex modulus in phase-separated poly (lactic acid)/poly (ethylene oxide)/carbon nanotubes nanocomposites. Polym. Test. 2018, 66, 189-194. [CrossRef]

27. Zare, Y.; Rhee, K.Y. A multistep methodology for calculation of the tensile modulus in polymer/carbon nanotube nanocomposites above the percolation threshold based on the modified rule of mixtures. RSC Adv. 2018, 8, 30986-30993. [CrossRef]

28. Zare, Y.; Rhee, K.Y. Tensile modulus prediction of carbon nanotubes-reinforced nanocomposites by a combined model for dispersion and networking of nanoparticles. J. Mater. Res. Technol. 2020, 9, $22-32$. [CrossRef]

29. Favier, V.; Chanzy, H.; Cavaille, J. Polymer nanocomposites reinforced by cellulose whiskers. Macromolecules 1995, 28, 6365-6367. [CrossRef]

30. Zare, Y.; Rhee, K.Y. Study on the Effects of the Interphase Region on the Network Properties in Polymer Carbon Nanotube Nanocomposites. Polymers 2020, 12, 182. [CrossRef]

31. Zare, Y.; Rhee, K.Y.; Park, S.-J. Modeling the roles of carbon nanotubes and interphase dimensions in the conductivity of nanocomposites. Results Phys. 2019, 15, 102562. [CrossRef]

32. Sisakht Mohsen, R.; Saied, N.K.; Ali, Z.; Hosein, E.M.; Hasan, P. Theoretical and experimental determination of tensile properties of nanosized and micron-sized CaCO3/PA66 composites. Polym. Compos. 2009, 30, 274-280. [CrossRef]

33. Fornes, T.; Paul, D. Modeling properties of nylon 6/clay nanocomposites using composite theories. Polymer 2003, 44, 4993-5013. [CrossRef]

34. Peng, W.; Rhim, S.; Zare, Y.; Rhee, K.Y. Effect of " $Z$ " factor for strength of interphase layers on the tensile strength of polymer nanocomposites. Polym. Compos. 2019, 40, 1117-1222. [CrossRef]

35. Chen, S.; Sarafbidabad, M.; Zare, Y.; Rhee, K.Y. Estimation of the tensile modulus of polymer carbon nanotube nanocomposites containing filler networks and interphase regions by development of the Kolarik model. RSC Adv. 2018, 8, 23825-23834. [CrossRef]

36. Zare, Y.; Rhee, K.Y. Tensile modulus of polymer/CNT nanocomposites containing networked and dispersed nanoparticles. AIChE J. 2018, 64, 220-225. [CrossRef]

37. Baxter, S.C.; Robinson, C.T. Pseudo-percolation: Critical volume fractions and mechanical percolation in polymer nanocomposites. Compos. Sci. Technol. 2011, 71, 1273-1279. [CrossRef] 
38. Celzard, A.; McRae, E.; Deleuze, C.; Dufort, M.; Furdin, G.; Marêché, J. Critical concentration in percolating systems containing a high-aspect-ratio filler. Phys. Rev. B 1996, 53, 6209. [CrossRef]

39. Zare, Y.; Rhee, K.Y. A multistep methodology for effective conductivity of carbon nanotubes reinforced nanocomposites. J. Alloys Compd. 2019, 793, 1-8. [CrossRef]

40. Loos, M.; Manas-Zloczower, I. Micromechanical models for carbon nanotube and cellulose nanowhisker reinforced composites. Polym. Eng. Sci. 2013, 53, 882-887. [CrossRef]

41. Chatterjee, A.P. A model for the elastic moduli of three-dimensional fiber networks and nanocomposites. J. Appl. Phys. 2006, 100, 054302. [CrossRef]

42. Ouali, N.; Cavaillé, J.; Perez, J. Elastic, viscoelastic and plastic behavior of multiphase polymer blends. Plast. Rubber Compos. Process. Appl. (UK) 1991, 16, 55-60.

43. Pukanszky, B. Influence of interface interaction on the ultimate tensile properties of polymer composites. Composites 1990, 21, 255-262. [CrossRef]

44. Lazzeri, A.; Phuong, V.T. Dependence of the Pukánszky's interaction parameter B on the interface shear strength (IFSS) of nanofiller-and short fiber-reinforced polymer composites. Compos. Sci. Technol. 2014, 93, 106-113. [CrossRef]

45. Szazdi, L.; Pozsgay, A.; Pukanszky, B. Factors and processes influencing the reinforcing effect of layered silicates in polymer nanocomposites. Eur. Polym. J. 2007, 43, 345-359. [CrossRef]

46. Callister, W.D.; Rethwisch, D.G. Materials Science and Engineering: An Introduction; Wiley: New York, NY, USA, 2007.

47. Durmus, A.; Kaşgöz, A.; Macosko, C.W. Mechanical Properties of Linear Low-density Polyethylene (LLDPE)/clay Nanocomposites: Estimation of Aspect Ratio and İnterfacial Strength by Composite Models. J. Macromol. Sci. Part B Phys. 2008, 47, 608-619. [CrossRef]

48. Zare, Y.; Daraei, A.; Vatani, M.; Aghasafari, P. An analysis of interfacial adhesion in nanocomposites from recycled polymers. Comput. Mater. Sci. 2014, 81, 612-616. [CrossRef]

49. Crosby, A.J.; Lee, J.Y. Polymer nanocomposites: The "nano" effect on mechanical properties. Polym. Rev. 2007, 47, 217-229. [CrossRef]

50. Li, D.; Kaner, R.B. How nucleation affects the aggregation of nanoparticles. J. Mater. Chem. 2007, 17, $2279-2282$. [CrossRef]

51. Zhou, Z.; Sarafbidabad, M.; Zare, Y.; Rhee, K.Y. Prediction of storage modulus in solid-like poly (lactic acid)/poly (ethylene oxide)/carbon nanotubes nanocomposites assuming the contributions of nanoparticles and interphase regions in the networks. J. Mech. Behav. Biomed. Mater. 2018, 86, 368-374. [CrossRef]

52. Zare, Y.; Rhim, S.; Garmabi, H.; Rhee, K.Y. A simple model for constant storage modulus of poly (lactic acid)/poly (ethylene oxide)/carbon nanotubes nanocomposites at low frequencies assuming the properties of interphase regions and networks. J. Mech. Behav. Biomed. Mater. 2018, 80, 164-170. [CrossRef] [PubMed]

53. Frankland, S.; Caglar, A.; Brenner, D.; Griebel, M. Molecular simulation of the influence of chemical cross-links on the shear strength of carbon nanotube-polymer interfaces. J. Phys. Chem. B 2002, 106, 3046-3048. [CrossRef]

54. Isitman, N.A.; Aykol, M.; Kaynak, C. Nanoclay assisted strengthening of the fiber/matrix interface in functionally filled polyamide 6 composites. Compos. Struct. 2010, 92, 2181-2186. [CrossRef]

55. Jamalzadeh, N.; Heidary, S.; Zare, Y.; Rhee, K.Y. A multistep methodology based on developed Takayanagi, Paul and Ouali models for tensile modulus of polymer/carbon nanotubes nanocomposites above percolation threshold assuming the contribution of interphase regions. Polym. Test. 2018, 69, 1-8. [CrossRef]

56. Zou, W.; Du, Z.-J.; Liu, Y.-X.; Yang, X.; Li, H.-Q.; Zhang, C. Functionalization of MWNTs using polyacryloyl chloride and the properties of CNT-epoxy matrix nanocomposites. Compos. Sci. Technol. 2008, 68, 3259-3264. [CrossRef]

(C) 2020 by the authors. Licensee MDPI, Basel, Switzerland. This article is an open access article distributed under the terms and conditions of the Creative Commons Attribution (CC BY) license (http://creativecommons.org/licenses/by/4.0/). 